\title{
La Facultad de Humanidades \\ en la cárcel: formación docente, investigación y extensión
}

\section{Brenda Hidalgo}

Docente investigadora de la Facultad de Humanidades. Universidad Nacional de Catamarca - Consejo Nacional de Investigaciones Científicas y Técnicas (CONICET), Argentina.

brenda.hgo@hotmail.com

\section{Mario Arnoldo Alanís}

Docente investigador de la Facultad

de Humanidades. Universidad

Nacional de Catamarca

maalanis@arnet.com.ar
Integración de la docencia y la extensión /

Intervenciones

RECEPCIÓN: 24/06/16

ACEPTACIÓN FINAL: 10/10/16

\section{Resumen}

Las prácticas de extensión universitaria han permitido la construcción de vínculos entre actividades académicas y la comunidad local y se han establecido líneas de acción que apuestan al fortalecimiento de políticas de inclusión mediante la creación de programas, los cuales promueven el desarrollo de proyectos de investigación y la realización de propuestas interdisciplinarias con criterios de participación y responsabilidad entre las partes componentes. En el presente trabajo se propone dar a conocer algunos de los desempeños en el marco del programa "La Facultad de Humanidades en la cárcel", perteneciente a la Universidad Nacional de Catamarca. Consideramos que tales actividades han permitido, tanto en la formación como en la investigación, generar procesos que nos sitúan frente a una función social y política de fortalecer espacios que garanticen la educación como un derecho social, trascendiendo contextos.

Palabras-clave

- Extensión

- Formación docente

- Investigación

- Cárcel

\section{Resumo}

As práticas de extensão universitária têm permitido a criação de vínculos entre atividades acadêmicas e a comunidade local, estabelecendo-se linhas de ação que confiam no fortalecimento das políticas de inclusão através da criação de programas que promovem o desenvolvimento de projetos de pesquisa e a realização de propostas interdisciplinares, com critérios de participação e responsabilidade entre as partes componentes. Neste trabalho propomo-nos apresentar alguns dos desempenhos obtidos no âmbito do programa "La Facultad de Humanidades en la Cárcel”, pertencente à Universidade Nacional de Catamarca. Acreditamos que tais atividades têm permitido tanto na formação quanto na pesquisa, gerar processos que nos situam diante da função social e política de fortalecer espaços que garantam a educação como un direito social, transcendendo contextos.

Palavras-chave

- Extensão

- Formação docente

- Pesquisa

- Presídio
Para citación de este artículo

Hidalgo, B. y Alanís, M. A. (2016). La Facultad

de Humanidades en la cárcel: formación docente, investigación y extensión. En Revista +E versión digital, (6), pp. 446-452. Santa Fe, Argentina: Ediciones UNL. 


\section{El programa en marcha}

En el año 2005 se puso en marcha el programa de extensión:

"La Facultad de Humanidades en la cárcel" con la intención de institucionalizar acuerdos con el Servicio Penitenciario de la Provincia de Catamarca. Tal implementación buscó, por un lado, acercar el dictado y conocimiento de la oferta académica al interior de la cárcel y, por otro, generar espacios de apertura a tareas vinculadas con el desarrollo de proyectos de investigación. Ante ello, entre los propósitos ${ }^{1}$ con los que cuenta la propuesta, podemos mencionar:

- Contribuir al dictado de materias de carreras que ofrece la Facultad de Humanidades, en el ámbito del Servicio Penitenciario Provincial (SPP) y que tenga como destinatarios a privados de libertad, quienes por sus condiciones de detención no pudieran asistir a la sede de la unidad académica.

- Fomentar el desarrollo de proyectos de investigación que contribuyan a producir conocimientos sobre la construcción de identidades, las prácticas cotidianas, códigos, trayectorias sociales, entre otros aspectos, al interior del Servicio Penitenciado de la provincia.

- Orientar los medios y proveer los recursos que permitan la ejecución de proyectos de extensión aprobados por el Consejo de Extensión de la Facultad de Humanidades y que tenga como destinatarias a las personas recluidas en la cárcel.

1) Los objetivos han sido retomados del marco acuerdo emitido entre la Facultad de Humanidades y el Servicio Penitenciario de la Provincia, bajo la resolución C.D.F.H.N 050. Dando origen al programa: La Facultad de Humanidades en la Cárcel. 2) Desde el año 2008, la Unidad Penal de Varones $n^{\circ} 3$, se encuentra en el nuevo edificio del SPP, ubicado en Miraflores, departamento Capayán, a kilómetros de la ciudad capital. 3) Hasta el año 2014, funciono la Uni- dad correccional de Mujeres en la calle 1 de Mayo, actualmente se encuentra ubicado cerca del antiguo predio del SPP, por Av. Colón y Mendoza. 4) Hasta el año 2007 se ubicaba en la ciudad capital, sobre Avenida Güemes y Avenida Virgen del Valle. 5) Están mediadas por docentes, egresados, alumnos avanzados y ayudantes de cátedras. Al momento de ingresar a la cárcel se emite una nomina con los datos y dedicación correspondiente de los integrantes del

En el marco de las responsabilidades institucionales, entre la Facultad de Humanidades y el SPP, se establecen acuerdos vinculados, a cargo de esta última institución, relacionados con el traslado de las personas responsables del dictado de clases en el penal de varones, el cual se encuentra ubicado a varios kilómetros de la ciudad, ${ }^{2}$ en tanto que el penal de mujeres se sitúa a unas cuadras de la Universidad. ${ }^{3}$ Asimismo, el SPP se encarga del traslado de los alumnos/as que obtienen el permiso para cursar sus estudios en la Facultad.

En sus primeros años, el desarrollo de las actividades académicas se llevo a cabo mediante la participación voluntaria de docentes y egresados de distintos departamentos: Trabajo Social, Inglés, Filosofía, Ciencias de la Educación, Geografía e Historia. Ellos asistían al SPP ${ }^{4}$ para promover el acceso de las personas que se encontraban privadas de su libertad a las distintas carreras de la Facultad. En los últimos años, la intervención se extendió a otros claustros, por lo que en la actualidad se desempeñan en el dictado de materias también ayudantes alumnos y alumnos avanzados. Las tareas académicas que se inician ${ }^{5}$ mediante el dictado de los talleres de orientación vocacional y vida universitaria son las primeras acciones que se llevan a cabo y no solo permiten conocer la participación de los alumnos/as detenidos/as inscriptos en cada carrera $^{6}$ sino que se generan instancias de resignificación de los

programa y proyectos de investigación, para dar inicio al dictado de las clases y trabajos de investigación, tanto en el penal de varones como en la cárcel de mujeres.

6) La nomina de alumnos/as que asisten a clases es autorizada por el juez de Instrucción Penal con asesoramiento del equipo profesional del SPP. Es enviada a la Facultad de Humanidades. En este sentido, al interior de la cárcel, se plantea la convivencia de lógicas de funcionamiento que se enmarcan, por un lado, desde la penitenciaria, en los sistemas normativos de ejecución de la Pena Privativa de la Libertad (Ley 24660), y por otro, desde la Universidad, en el hacer autónomo de sus prácticas y la promoción de sus políticas educativas, las cuales se emparentan con los propósitos que trae la Ley de Educación Nacional (26206) al buscar la restitución de derechos educativos en tales contextos. 
procesos de formación ante estos nuevos escenarios de desempeño. Las actividades al interior de los penales se realizan en los espacios establecidos para el funcionamiento educativo. De ese modo, tanto en el penal de varones ${ }^{7}$ como en el penal de mujeres ${ }^{8}$ se cuenta con un lugar destinado a ello. No obstante, la disponibilidad de aulas es más amplia en la de varones, por lo que se pueden desarrollar varias clases al mismo tiempo. En el caso de las mujeres, al tener un aula de usos múltiples, el tiempo prevalece en la designación de las actividades por realizar.

El reconocimiento de tales tareas académicas tiende a construir un lugar de afirmación de los derechos educativos de las personas que se encuentran cumpliendo una condena o están procesadas, de manera que en su horizonte emerge el sistema universitario como una posibilidad.

Desde este posicionamiento, estos contextos de privación de la libertad se establecen en espacios sociales permeables y la aproximación al interior de la cárcel permite generar otras maneras de relacionamiento, nuevas miradas en torno al contexto como lugar de construcción de prácticas de enseñanza-aprendizaje, de investigación y formación docente, que van más allá de los dispositivos de seguridad propios del lugar. Siendo la cárcel pensada como un espacio que no solo debe ser reducida a mecanismos de vigilancia y control, donde, si bien prevalece el binomio de lo permitido y lo prohibido, también es factible que sea un lugar de producción de significados y otorgamiento de sentido al interior de ese ámbito, donde se busca construir espacios de apertura, de diálogo y de interrogantes con relación al sentido que se va construyendo entorno a la educación.

Partiendo de un enfoque que busca generar instancias de prácticas que se apartan de posturas vinculadas a la acción e intervención, como campo de aplicación penitenciaria, ${ }^{9}$ como dispositivo de funcionamiento, que condiciona y regula la vida total de los sujetos. Consideramos que aprender es aprehender desde la pregunta, desde la ejemplificación, desde la representación de situaciones que permiten pensar, repensar y reflexionar más allá de la mera transmisión de contenidos. Este posicionamiento es compartido con el planteo que realiza Nuñez Pérez (2009:67), quien sostiene que:

"En la acción educativa no se trata de imperativos, sino de tiempos disponibles para escuchar y ser escuchados; para preguntar y preguntarse... para ver pasar otros mundos y albergar en ellos esperanzas otras. Dar lugar a otras miradas, a otras maneras de entender el mundo, de explicarlo y explicarse".

\section{Acciones vinculadas a la formación docente}

En este marco emergen cuestiones vinculadas a los sentidos que asumen las prácticas de enseñanza-aprendizaje en estos contextos, que se convierten en escenario que siguen siendo poco visibles en los procesos de formación de educación superior universitaria, por lo cual podemos preguntarnos:¿cómo se integra esta modalidad de enseñanza en los aprendizajes curriculares de los estudiantes?, ¿se piensa en estos escenarios como espacios de desempeño profesional?, ¿qué demandas y desafíos generan en la formación docente?

La problematización sobre el lugar que adquieren estos contextos, en la formación docente inicial, en algunos casos, dependen de posicionamientos y lineamientos políticos-académicos que, pueden ser asumidos mediante la incorporación de contenidos curriculares que den a conocer los propósitos y objetivos de las modalidades educativas, en este caso puntual, nos referimos a la educación en contextos de privación de la libertad; no obstante, una posible respuesta a la incorporación de estos escenarios se genera, a través de actividades de extensión, de propuestas de formación, que permiten incorporar ésta modalidad, en el desempeño de tareas que se construyen desde practicas situadas.

Ante ello, consideramos que las instancias de prácticas de enseñanza, que se llevan a cabo al interior de la cárcel, amplían los escenarios pedagógicos, poniendo de manifiesto estos espacios como posibles lugares de desempeño profesional, que poco son visibilizados. La construcción de práctica, en este sentido, está vinculado al planteo que realiza Alliaud y Antelo (2011), al establecerse como un puente entre la institución formativa y las instituciones escolares, siendo en los contextos de actuación donde los alumnos empiezan a transitar nuevos roles y desempeños, del mismo modo que emergen demandas y desafíos que provienen en confrontación con elementos que se van adquiriendo en la formación.
7) Funciona la Escuela de Adultos $n^{\circ} 43$ (primaria-alfabetización), CENS $n^{\circ} 50$ (secundaria).

8) Funciona el anexo del CENS $n^{\circ} 50$ (secundaria).

9) Se alude a la concepción de educa- ción enmarcada como tratamiento, como practica de reinserción, reeducación y resocialización. Estudios sobre ello se encuentra en Daroqui (2000), Gutiérrez (2010), Herrera (2010). 


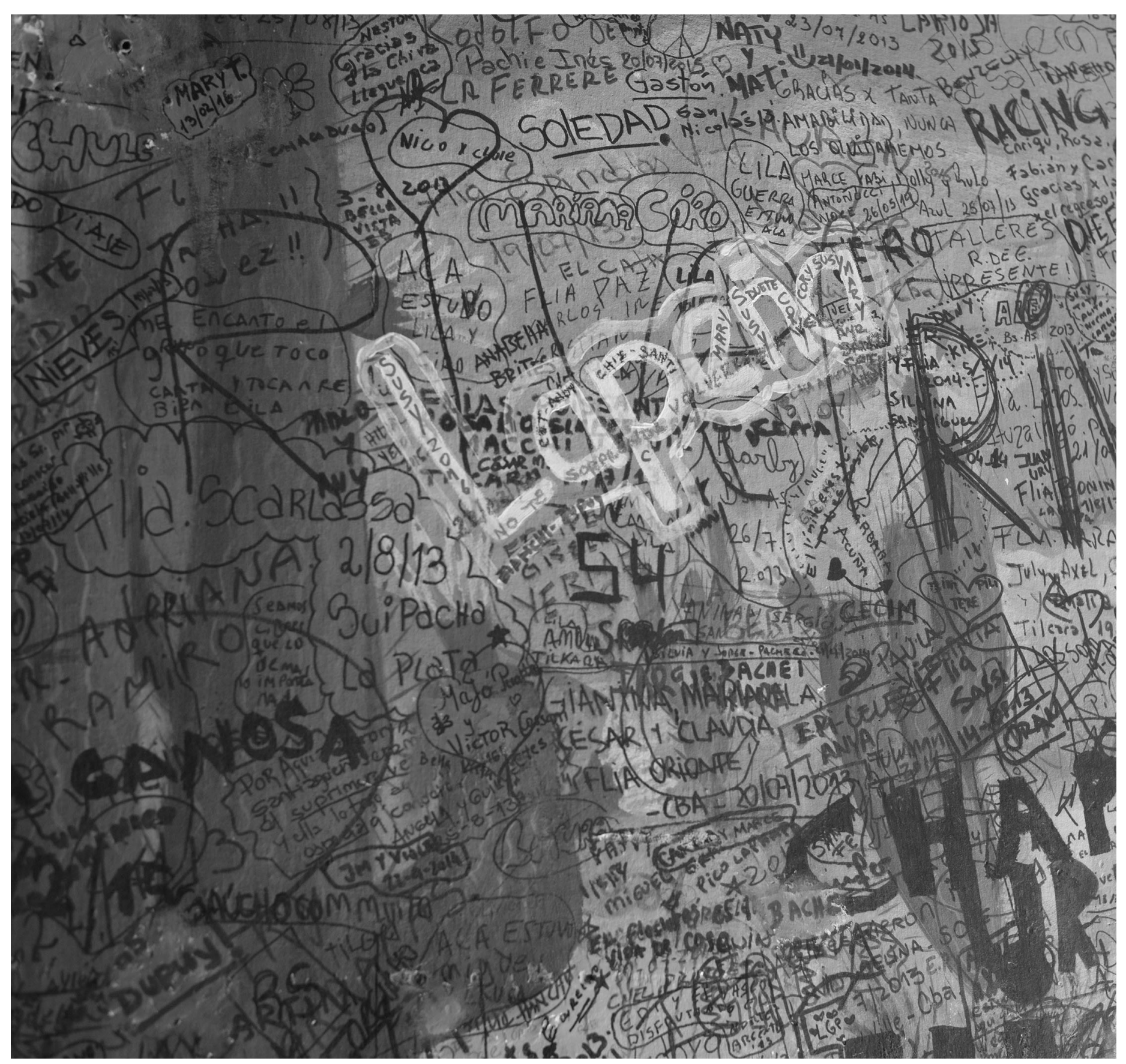

(๑) Hugo Pascucci 


\section{6}

\author{
creemos que la formación docente, debe \\ ser capaz de generar instancias de reflexión \\ en pos de desarmar un discurso arraigado \\ sólo en la noción de enseñanza
}

Resulta importante señalar que estas demandas están vinculadas a las construcciones significativas que se original, en los contextos de desempeño real, ante demandas institucionales, que llevan a problematizar la propia experiencia, con los saberes curriculares. En este sentido, como plantea Schon (citado en Sanjurjo, 2014), la reflexión en la acción y la reflexión sobre la acción nos permiten construir conocimiento sobre lo que estamos haciendo mientras lo estamos haciendo. En otras palabras, lleva a replantear el proceso de enseñanza continuamente en función de brindar herramientas necesarias de aprendizaje, respondiendo a la multiplicidad y complejidad de los contextos de actuación.

De ese modo, el trabajo a través de encuentros, con alumnos avanzados y ayudantes de cátedras, que participan del programa, nos permitió ir trabajando en forma conjunta en la construcción de tres dimensiones, las cuales son retomadas de los aportes de Huergo y Morawichi (2008):

- El espacio diseñado: ante el reconocimiento del espacio y la organización de los tiempos (ubicación de la escuela al interior del penal): prescripciones y regulaciones establecidas.

- El espacio recorrido: en el desarrollo de algunas actividades, al momento de esbozar estrategias didácticas, aludiendo a las inscripciones particulares de lo vivenciado en el hacer de las prácticas.

- El espacio representado: se trabaja con relación a las dificultades de aprendizajes que se presenta en los/as estudiantes preso/as, vinculadas al material de estudio, interpretación y análisis de los textos, la atención y participación, siendo indicadores que llevan a trabajar en la restructuración y planificación de las clases, al momento de pensar cada encuentro. Los cuales adquieren sentidos diferentes y ante los cuales se regresa con sensaciones diferentes (lo percibido y lo significado) (Huergo y Morawicki 2008).

Poder pensar estas categorías teóricas, es un modo de indagar en la formación de prácticas preprofesionales, que les permiten, por un lado, una movilidad itinerante, donde de ser alumnos pasan a desempeñar tareas que los ubica ante posicionamientos y maneras de actuar, custodiadas bajo lógicas instituidas de la institución escolar, y por otro, otorgar sentido y significado a la educación en estos contextos de privación de la libertad.

En esta etapa de formación, el aprendizaje colaborativo y participativo, es la impronta con la que se ha venido trabajando, desde el programa, a lo largo de todos estos años. Ante ello, creemos que la formación docente, debe ser capaz de generar instancias de reflexión en pos de desarmar un discurso arraigado sólo en la noción de enseñanza, para poder añadir otros componentes que la integran: sujetos, contextos, realidades, vivencias, experiencias. De ese modo, la relación pedagógica no se establece desde relaciones verticales, sino que se construye a través de vínculos e instancias de relacionalidad donde emerge el desdibujamiento de escenas de prejuicios condenatorios hacia quienes están procesados/as o condenados/as.

La enseñanza mediada por el diálogo, la interacción, ejemplificación y problematización, ante las exigencias del contexto, es un pilar que sostiene las prácticas docentes que se construyen al interior de la cárcel. En aquel mundo complejo, susceptible de interpretaciones, representaciones, estigmas y desconocimiento. Poder trascender los barrotes y muros, los imaginarios y los supuestos sobre la cárcel, es lo que ha permitido y sigue permitiendo poder llevar a cabo anualmente la coordinación de los grupos de trabajo que ingresan al interior de estos contextos.

Un aspecto importante de estas actividades ha sido generar instancias de intercambios y socialización a través de talleres, en jornadas y congresos, lo cual ha posibilitado el debate y la construcción de aprendizajes compartidos, pensando en la modalidad educativa de educación en contexto de privación de la libertad en la formación docente.

\section{Acciones desarrolladas vinculadas a la investigación}

La creación del Programa tendió un puente hacia el interior de la cárcel, como un ámbito de investigación, como un estudio social, que se aleja de su fisonomía clásica como dispositivo de castigo 
para ser pensado desde las acciones humanas acompañadas de sentidos y significados, que emergen de procesos de subjetivación e interpretación. De este modo, al margen del ámbito judicial y penal que impregnan estos contextos, nos situamos frente sujetos de derechos, de diálogos y conversaciones, sobre su estar allí. El primer trabajo de investigación que se llevo a cabo estaba conformado por docente y egresados que participaban del dictado de materias en el SPP. Entre los interrogantes que se formulaban estaba el interés puesto en las interacciones y prácticas que se van construyendo y reconstruyendo ante la convivencia y los procesos de socialización que se configuran al interior de la cárcel. Así, en: "Estudios sobre la cárcel: una aproximación a las prácticas y estrategias de constitución y reproducción de identidades individuales y colectivas (2005-2008)", ${ }^{10}$ se indago respecto de las formas de relacionarse; el sentido y el significado que se les asignan a esa situación de privación; la concepción y uso del espacio y el tiempo en la cárcel. Ante esto último se tuvo en cuenta la participación en la investigación de guardiacárceles, en pos de poder aproximarse a las prohibiciones y permisos que circulan al interior. Con relación al ámbito educativo, estas investigaciones permitieron comenzar e indagar la cárcel como un nuevo contexto de enseñanza-aprendizaje.

Desde el año 2009 se desarrolla un nuevo proyecto de investigación denominado: "La vida más allá del encierro: las prolongaciones de la sociedad en la organización de la vida comunitaria en la cárcel", ${ }^{11}$ y en el mismo participan alumnos becarios y alumnos avanzados de distintas carreras de la Facultad de Humanidades, generándose nuevas dinámicas de trabajo para la promoción e integración de los aprendizajes, experiencias y conocimientos producidos por ellos. El eje organizador de esta investigación se centraba en la unidad penal de varones. Su indagación partía de la reciente reubicación, que se produjo en el año 2008, del nuevo edificio del Sistema Penitenciario. Se buscaba conocer el impacto que generaba la nueva ubicación del penal, con relación a los vínculos que se establecen entre los presos con sus familiares y demás agentes externos. La nueva estructura y organización de los pabellones al interior de la cárcel establecían nuevos interrogantes que llevaron a preguntarse respecto de las relaciones sociales que se generaban entre los presos, es decir, prácticas y significaciones resultado de sus estrategias de socialización. Pensando ello en cuanto al espacio carcelario y sus prescripciones, reglamentaciones y mecanismos de regulación. Respecto a esto último se trabajo con relación a la cárcel de mujeres. Con relación al ámbito educativo, el lugar de la escuela y los procesos de escolarización cobraban vital importancia. Desde el año 2014 se está trabajando con algunos interrogantes que se manifiestan en la formulación del proyecto: "Sociedad y delito en Catamarca. Recorridos delictivos, políticas punitivas y prácticas culturales al interior de la cárcel". ${ }^{12}$ La pretensión de esta investigación es profundizar en la situación de las mujeres que se encuentran procesadas y detenidas en la cárcel de mujeres, y seguir ahondando en el conocimiento de las relaciones y vínculos que se vienen trabajando sobre el penal de varones. La característica de este proyecto se da en la participación interinstitucional con miembros integrantes que se desempeñan en la Facultad de Derecho de la Universidad Nacional de Catamarca. Este trabajo en forma conjunta ha llevado a que los interrogantes de la investigación no solo sean planteados desde las ciencias sociales sino que se aúna en su problematización el campo del Derecho. Los proyectos desarrollados en tales contextos se han caracterizado por el trabajo interdisciplinario y relacional que aportan los integrantes ante distintas miradas, posicionamientos y enfoques desde sus campos de formación. Asimismo, la reconfiguración de las dinámicas de funcionamiento ha llevado a la incorporación de alumnos de las distintas carreras, generándose una ampliación ante la participación de prácticas de investigación como campo de formación. En algunos casos, estos procesos formativos han sido impulsados por políticas promovidas por becas de investigación ${ }^{13}$ que fortalecen el acompañamiento en el desarrollo de los primeros desempeños como la continuidad y formación de recursos en estos ámbitos.

El intercambio de opiniones y posicionamientos con relación a la cárcel como ámbito de estudio ha generado la producción de
10) Proyecto acreditado por la Sedecyt UNCA (2005-2008) a cargo del Mgter. Alanís, Mario Arnoldo; integrado por: Bértola, Leonardo; Herrera Herminda; Narváez, Gabriela; Pessacq María Isabel;

Jalile, Eleonora; Cardoso, Ana del Huerto; Gordillo, Silvana; Pistarelli, Mariela. 11) Proyecto acreditado por la SECYT UNCA (2009-2012) a cargo del Mgter. Alanís, Mario Arnoldo; integrado por: Cardoso, Ana del Huerto; Narváez,
Gabriela; Gordillo, Silvana; Iturriza, Joaquín; Orellana, Paola; Zurita, Claudia; Mensa, Cecilia; alumnos: Córdoba, Nancy; Hidalgo, Brenda. 12) Proyecto acreditado por la SECYT UNCA (2014-2017) a cargo del Mgter. Alanís, Mario Arnoldo; integrado por: Cardoso, Ana del Huerto; Orellana, Paola; Vaquinsay, Elizabeth; Tarcaya, Carina; Hidalgo, Brenda; Torres, Nelson; Lilljedalh, Enrique; Carrizo Rubén; Vera, Mariana; alumnos:
Miranda, Melisa; Gómez, María. 13) El programa de becas emitidas por el Consejo Interuniversitario Nacional $(\mathrm{CIN})$ ha promovido la participación e interés responsable de alumnos que han iniciado sus primeros pasos en la investigación a través del desarrollo de un plan de trabajo que se va realizando mediante el acompañamiento y participación en las distintas actividades llevadas a cabo por el trabajo en equipo con los miembros integran- tes del proyecto de investigación. Asimismo, la participación en estos proyectos ha permitido un espacio para el trabajo de egresados que, a través del otorgamiento de becas promovidas por el Consejo Nacional de Investigaciones Científicas y Técnicas (CONICET), pueden seguir fortaleciendo los conocimientos y aportes que se generan desde la investigación como campo de formación. 


\section{4}

\author{
esas voces acalladas son las que irrumpen \\ en las narrativas, entrevistas, en charlas \\ y diálogos que se tienen con esos Otros \\ que son parte de un nos-otros social
}

conocimientos puestos a consideración a través de una serie de publicaciones denominada: La vida en la cárcel. Intercambios, exclusión y control social. El trabajo de estas publicaciones no solo remite a teorías, marcos epistemológicos y estrategias metodológicas, sino que da lugar a aquellas voces que son, sentenciadas y condenadas socialmente, como cuerpos depositados en un lugar que debe mantenerlos alejados, olvidados e invisibilizados, emergiendo en los discursos ante el miedo y la estigmatización. Esas voces acalladas son las que irrumpen en las narrativas, entrevistas, en charlas y diálogos que se tienen con esos Otros que son parte de un nos-otros social.

\section{A modo de cierre e integración}

Las tareas de investigación y docencia que se generan desde el Programa de extensión "La Facultad de Humanidades en la cárcel" han constituido procesos que fortalecen políticas de inclusión y de interés ante los derechos educativos de las personas que se encuentran en contextos complejos como la cárcel. Desde la Facultad de Humanidades, tales prácticas han sido un anclaje con aquellos ámbitos de la vida social que son interpelados por las acciones y determinaciones de discursos que remiten al ámbito jurídico-penal. La cárcel se ha constituido en un lugar de aprendizaje, posibilitando el trabajo interdisciplinario, interclaustro e interinstitucional, que se va articulando al momento de debatir, pensar y repensar las tareas que se llevan a cabo, y haciendo factibles instancias de trabajo que materializan, desde múltiples miradas, la producción de conocimientos.

El trabajo colaborativo ha sido, y sigue siendo, un pilar fundamental en el desarrollo de esta tarea, la cual, en términos de políticas educativas, acompaña en la divulgación y conocimiento de la modalidad educativa: Educación en contextos de privación de libertad, regulada por la Ley de Educación Nacional 26206, y promueve el acceso, la integración y la permanencia de alumnos/as detenido/as al sistema de formación de estudios superiores.
La creación de programas que dan visibilidad a contextos donde el proceso de formación se integra desde la formación docente, la investigación y la extensión, genera instancias de aprendizajes que permiten interpelar posicionamientos, prácticas y supuestos que se construyen y de-construyen ante acciones que involucran la responsabilidad, el compromiso y el deseo de llevar a cabo estas tareas.

\section{Referencias bibliográficas}

Alliaud, A. y Antelo, E. (2011). Los gajes del oficio. Enseñanza, pedagogía y formación. Buenos Aires: Aique.

Daroqui, A (2000) "La cárcel en la universidad. El discurso penitenciario en la normativa y prácticas interinstitucionales". En Nari, M y Fabre, A (comp) (2000) Voces de mujeres encarceladas. Buenos Aires, ed. Catálogos.

Gutiérrez, M (2010) "Derechos y sistema penal: la dimensión jurídica de las prácticas en contextos de encierro". En Herrera, P y Frejtman, V (2010) Pensar y hacer educación en contextos de encierro. Buenos Aires: Ministerio de Educación de la Nación. Huergo, J. y Morawicki K. (2008). Re-leer la escuela para re-escribirla (I) La escuela como espacio social. En Dirección Provincial de Educación Superior y Capacitación Educativa Dirección de Educación Superior Subdirección de Formación Docente. Disponible en: http://www.formadores.org/default_archivos/comunicados2009modulopractica.pdf

Ministerio de Educación, Ciencia y Tecnología de la Nación (2006). Ley 26206 de Educación Nacional. Hacia una Educación de calidad para una sociedad más justa. Núñez Pérez, V. (2010). Espacios carcelarios/espacios educativos. En T. Añaños,

F. (Coord.), Las mujeres en las prisiones. La educación Social en contextos de riesgo y conflicto (pp. 65-75). Barcelona: Gedisa.

Sanjurjo, L. (2014). Razones que fundamentan nuestra mirada acerca de la formación en las prácticas. Los dispositivos para la formación en las prácticas profesionales. Rosario: Homo Sapiens.

Universidad Nacional de Catamarca (9 de agosto de 2005). Resolución CDFH nº 050: Programa "La Facultad de Humanidades en la Cárcel". 